\title{
Sternal Resection and Reconstruction for Solitary Plasmacytoma of the Sternum: Case Report
}

\author{
Chang Woo Choi, M.D., You Kyeong Park, M.D., Hwa Kyun Shin, M.D., Jae Woong Lim, M.D., Keun Her, M.D. \\ Department of Thoracic and Cardiovascular Surgery, Soonchunhyang University Bucheon Hospital, Bucheon, Korea
}

\section{ARTICLE INFO}

Received July 29, 2020

Revised September 24, 2020

Accepted October 5, 2020

Corresponding author

Chang Woo Choi

Tel 82-32-621-5307

Fax 82-32-621-6950

E-mail tcvsccw@schmc.ac.kr

ORCID

https://orcid.org/0000-0002-8042-7447

\begin{abstract}
A 63-year-old patient was admitted with a sternal fracture and mass. On evaluation, most of the body of the sternum had been destroyed by a tumor. Radical resection of the sternum was performed and part of the major pectoral muscles adherent to the sternal tumor was also resected. The chest wall defect was reconstructed with mesh, bone cement, and a titanium rib plate system. Reconstruction with this method seemed to be an appropriate procedure to prevent instability of the chest wall.
\end{abstract}

Keywords: Sternum, Plasmacytoma, Rib plate, Case report

\section{Case report}

A 63-year-old man was admitted to thoracic and cardiovascular surgery department with anterior chest wall pain that had developed 2 years previously after a sternal fracture, was progressive, and was characterized by repeated fractures. A physical examination revealed a palpable mass in the sternal area that was unstable and tender. Chest computed tomography $(\mathrm{CT})$ revealed a $7.8 \times 5.6 \times 10.3-\mathrm{cm}$ soft-tissue attenuated mass with bony destruction of the sternum. A CT-guided needle biopsy revealed diffuse plasmacytic proliferation. The serum immunoglobulin level was within the normal range and a whole-body bone scan detected no metastatic lesions.

The pain was so severe that we decided to perform a radical resection. However, obtaining clear tumor margins would have required total sternal resection, which could cause instability of the reconstructed chest wall. Therefore, when designing the surgical procedure, postoperative radiotherapy was planned, and the target was relief of chest pain, postoperative thoracic stability, and resection of as much of the tumor as possible. Magnetic resonance imaging (MRI) was used to determine the resection range; it revealed a well-marginated, enhancing chest wall mass in the sternal body and costal cartilages $4-7$, without invasion of the heart or other adjacent organs (Fig. 1).
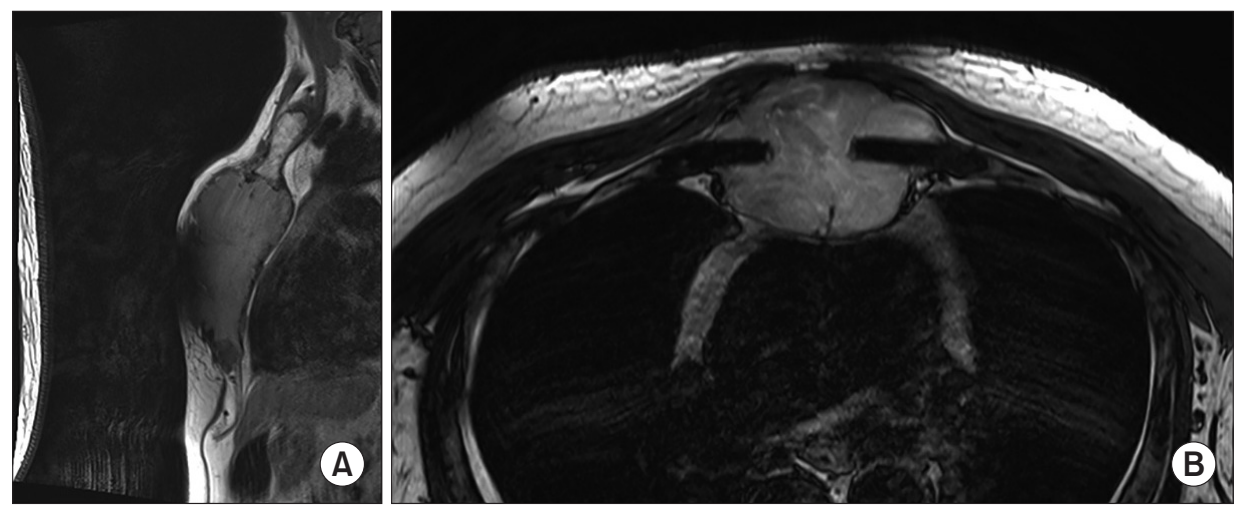

Fig. 1. (A, B) Chest magnetic resonance image shows a $7.8 \times 5.6 \times$ $10.3-\mathrm{cm}$ well-marginated, enhancing chest wall mass that was destroying the sternum.

Copyright (c) 2021, The Korean Society for Thoracic and Cardiovascular Surgery

(c) (i) \&) This is an Open Access article distributed under the terms of the Creative Commons Attribution Non-Commercial License (http://creativecommons.org/licenses/ by-nc/4.0) which permits unrestricted non-commercial use, distribution, and reproduction in any medium, provided the original work is properly cited. 
We decided that radical resection would include the entire sternum, except for the manubrium, parts of the pectoralis major muscles adherent to the sternal tumor, and costal cartilages 4-7. A rigid prosthetic reconstruction was necessary because of the wide resection planned, but no appropriate materials, such as titanium mesh, are available in our country to stabilize the anterior chest wall. We had previously performed sternal reconstruction in a sternal chondrosarcoma patient using Simplex antibiotic bone cement (Stryker, Mahwah, NJ, USA), which is usually used for arthroplasty; therefore, we decided to use Simplex again in this patient.

A wide vertical incision from the sternal notch to the xiphoid process was made with the patient in the supine position, and with the neck slightly extended. The skin and muscle layers were dissected to expose the whole sternum and costochondral junction. After retracting the muscle layers laterally, we resected parts of the pectoralis major muscles and costal cartilages 4-7. We transected the sternal body along the sternal angle, which provided a sufficient tumor-free margin. The tumor was dissected from the pericardium, aorta, and other inner organs. After removing the tumor, some of the anterior lymph nodes and pericardial fat were partially resected.

The liquid and bone cement powder were mixed and molded into the shape of a sternum for anterior chest wall reconstruction (Fig. 2). The thickness of the bone cement for the upper margin was determined based on the edge of the manubrium (where it met the bone cement). It was adjusted by making a frame and marking the depth before molding. Before placing the bone cement, we fixed macroporous polyester mesh (Parietex Flat Sheet Mesh; Covidien, Minneapolis, MN, USA) to both remaining ribs to protect the major vessels and pericardium. We then placed the bone cement over the mesh and fixed the titanium plate with screws to both remaining ribs and the manubrium

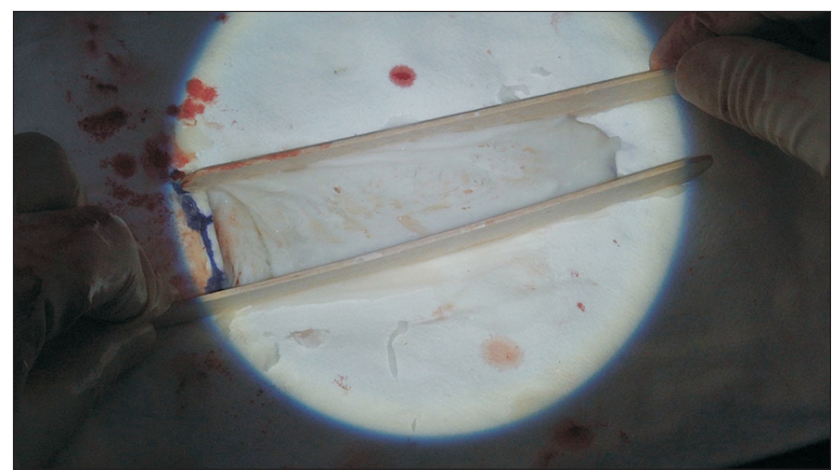

Fig. 2. Bone cement was molded into the shape of the sternum.
(Fig. 3). We first marked the molded bone cement where the screws would be placed. We used a drill to make holes slightly smaller than the screw in the marked locations, because the bone cement was too hard to insert the screws directly. We fixed the molded bone cement to the remaining manubrium first, and then fixed it to the ribs. These methods made screw insertion easy and stable. Last, the neosternum was covered with the pectoralis major muscle. One chest drain tube was placed posterior to the bone cement.

The patient recovered in the intensive care unit on postoperative day (POD) 1 and was moved to the general ward the next day. The chest tube was removed on POD 3, and the patient was discharged on POD 8 (Fig. 4). The pathological examination revealed dense plasmacytic proliferation, consistent with a solitary plasmacytoma. We resected the tumor with a $2-\mathrm{cm}$ gross margin through total resec-

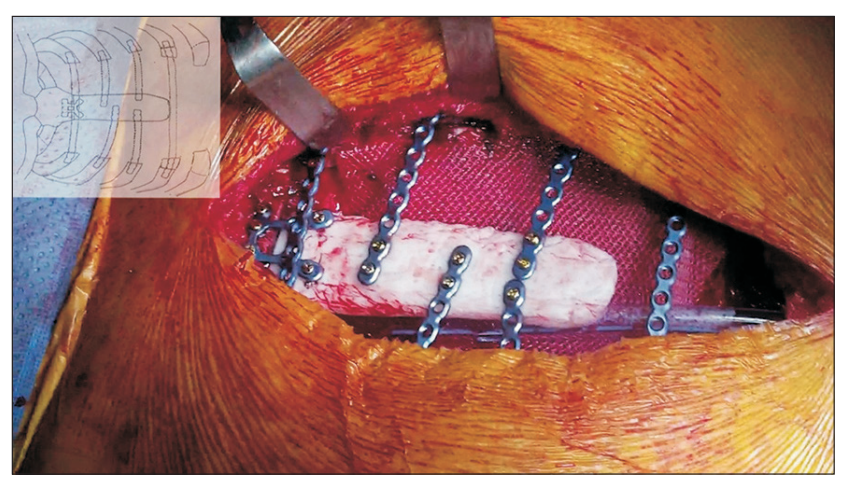

Fig. 3. Macroporous polyester mesh was placed; then, bone cement was placed over the mesh and fixed with a titanium plate.

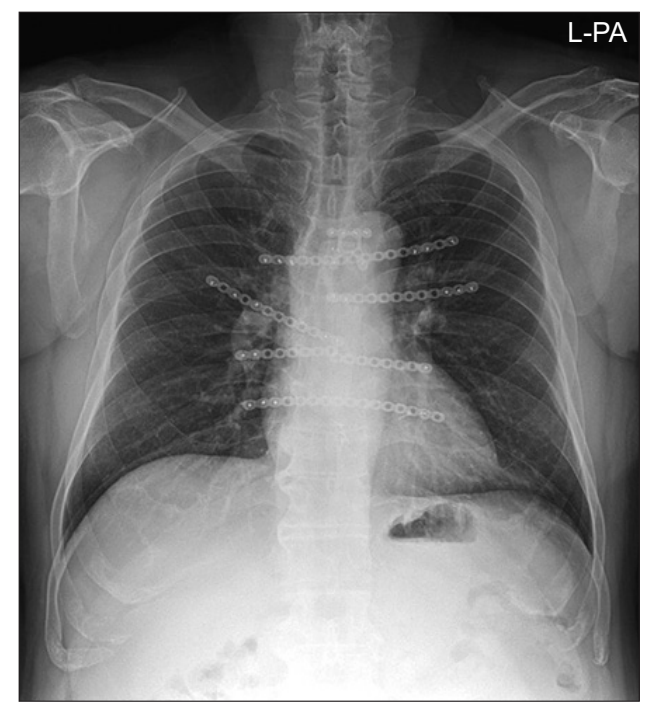

Fig. 4. Chest radiograph obtained 3 days postoperatively. 
tion of the sternum (except for the upper partial manubrium), but histologically, the clear resection margin was 0.7 $\mathrm{cm}$. The immunohistochemical analysis was positive for CD138 and monoclonal kappa light chains. After postoperative radiotherapy for 2 months, a contrast-enhanced chest CT scan revealed no evidence of recurrence. At the 6-month follow-up visit, there was no evidence of recurrence or any complications.

The patient provided written informed consent for the publication of his clinical details and images.

\section{Discussion}

Plasmacytoma refers to the neoplastic proliferation of a single clone of plasma cells, typically producing monoclonal immunoglobulin. Solitary plasmacytomas typically occur in bone, but can also be found in soft tissues (extramedullary plasmacytoma). A solitary plasmacytoma of bone is a localized tumor in the bone without features of multiple myeloma.

Radiotherapy is the treatment of choice for solitary plasmacytoma, while extensive resection is controversial. Radiotherapy would have been the treatment of choice for this patient if he did not have refractory chest pain. Wide excision is a treatment option for tumors that are completely resectable, and when there are tumor-induced symptoms such as severe pain, skeletal instability, or pathologic fracture. Wide excision is key for local control of a primary sternal tumor. Sternal resection with clear margins of at least $3 \mathrm{~cm}$ is advocated to minimize the risk of local recurrence. Treatment should be designed to address all manifestations of the disease shown by MRI and CT. Furthermore, localized radiotherapy should be administered even if the tumor is completely removed [1,2].

Reconstructing the sternum provides another challenge to the thoracic surgeon. A rigid prosthetic reconstruction is recommended for subtotal or total sternotomy. Sternal reconstruction has been traditionally performed with mesh and patches, with the addition of methyl methacrylate when rigidity is necessary.

The main goal of chest wall reconstruction is to eliminate dead space, restore skeletal rigidity, prevent lung herniation and impaction of the scapula, protect underlying organs, and maintain cosmesis. If full-thickness reconstruction is required, it must be determined whether the reconstruction should involve skeletal structures alone, or should be performed in conjunction with an overlying myocutaneous flap.

Various materials have been used to reconstruct the an- terior chest wall, including polypropylene mesh, titanium mesh, allogenic grafts, and three-dimensional printed titanium sternal and rib cages $[3,4]$. Titanium mesh is used for sternal defects $>5 \mathrm{~cm}$, without the need for additional autologous tissue. Due to its biocompatibility and flexibility, titanium mesh serves as a versatile and easy-to-implement polypropylene mesh to reconstruct sternal defects; however, its lack of rigidity may result in paradoxical chest wall motion [5].

No appropriate materials, such as titanium mesh, are available in our country for reconstructing the sternum. We propose that bone cement and titanium rib plates are a good substitute [6]. These materials are readily available and easy to handle, and the cement can be molded into the desired shape. The Simplex antibiotic bone cement we used contains $0.5 \mathrm{~g}$ of erythromycin and 3,000,000 IU of colistin, which makes the neosternum more resistant to infection. Regular follow-up visits are important to assess longterm results and complications, such as displacement of the neosternum, bone cement or titanium plate fracture, and infection. Macroporous polyester mesh has long-lasting tolerability, and can therefore protect the major vessels and pericardium from foreign-body reactions, and prevent fluid and air from moving between the pleural and subcutaneous spaces. The mesh functions as scaffolding for the regenerative connective tissue colonizing its surfaces, which also provides additional stability for the reconstructed chest wall [7].

In summary, bone cement with titanium rib plates can serve as a cost-effective and readily available alternative for sternum reconstruction.

\section{Conflict of interest}

No potential conflict of interest relevant to this article was reported.

\section{ORCID}

Chang Woo Choi: https://orcid.org/0000-0002-8042-7447

You Kyeong Park: https://orcid.org/0000-0002-8306-7627 Hwa Kyun Shin: https://orcid.org/0000-0003-2078-5098 Jae Woong Lim: https://orcid.org/0000-0003-4512-0236

Keun Her: https://orcid.org/0000-0001-7824-1988

\section{References}

1. Lee JH, Lee WS, Kim YH, Kim JD. Solitary plasmacytoma of the sternum. Korean J Thorac Cardiovasc Surg 2013;46:482-5. 
2. Chapelier AR, Missana MC, Couturaud B, et al. Sternal resection and reconstruction for primary malignant tumors. Ann Thorac Surg 2004;77:1001-6.

3. Aranda JL, Jimenez MF, Rodriguez M, Varela G. Tridimensional titanium-printed custom-made prosthesis for sternocostal reconstruction. Eur J Cardiothorac Surg 2015;48:e92-4.

4. Lee KH, Kim KT, Son HS, Jung JS, Cho JH. Porcine dermal collagen (permacol) for sternal reconstruction. Korean J Thorac Cardiovasc Surg 2013;46:312-5.
5. Krdzalic G, Mujagic H, Musanovic N, Krdzalic A. Titanium mesh reconstruction after solitary sternal plasmacytoma surgery: a case report. Int J Immunother Cancer Res 2017;3:41-3.

6. Jung JJ, Kim SS, Kang DH, et al. Sternal reconstruction with bone cement block using the rigid plate fixation technique. Thorac Cancer 2019;10:2022-5.

7. Thomas PA, Brouchet L. Prosthetic reconstruction of the chest wall. Thorac Surg Clin 2010;20:551-8. 